\title{
Organizational Culture Drives Confidence in Implementing Telestroke Care
}

\author{
Christopher Miao ${ }^{1}$, Holly Martin, MPH; ${ }^{2-3}$ Teresa Damush, PhD;2-4 Michelle LaPradd, MS; ${ }^{5}$ \\ Susan Ofner, MS; ${ }^{5}$ Linda Williams MD ${ }^{2,3,6}$ \\ ${ }^{1}$ Indiana University School of Medicine, ${ }^{2}$ Roudebush VA Medical Center; ${ }^{3}$ Regenstrief Institute, \\ Inc.; ${ }^{4}$ Indiana University School of Medicine, Department of Medicine; ${ }^{5}$ Indiana University \\ School of Medicine, Department of Biostatistics; ${ }^{6}$ Indiana University School of Medicine, \\ Department of Neurology
}

Background and Hypothesis: During the past two years, the Veteran Affairs' National Telestroke Program (NTSP) has conducted 800 teleconsultations at 30 participating sites. Site perceptions of the NTSP are collected in surveys at the time of the program entry and 6-12 months later. This study aims to determine factors associated with the confidence in providing stroke care among staff at participating hospitals, and whether confidence is associated with site performance on program quality indicators.

Experimental Design: Web-based surveys were sent to participating sites at baseline and post implementation. Survey questions pertained to a site's confidence providing stroke care, organizational readiness to change (ORCA assessment), buy-in of clinical services, and the impact of the program. Individual responses were averaged at each site. Confidence was scored from zero to ten and was dichotomized into fully confident (10) and not fully confident sites (<10). Site performance on key indicators of program quality were obtained from the NTSP data. A Kruskal Wallis analysis examined the association of baseline variables with postimplementation confidence.

Results: 54 individuals (57\% nurses, 26\% providers, and $7 \%$ administrators) from 16 sites had baseline and post-implementation data. Five sites were fully confident at the postimplementation assessment. The other 11 had confidence scores ranging from 8.32 to 9.5 . Higher ORCA scores were significantly associated with post-implementation confidence (mean ORCA 12.2 vs. $6.8, p=0.04$ ). Sites with longer NTSP participation were less likely to be fully confident, (mean 10.6 months vs. 3.8, $\mathrm{P}=0.01$ ). Baseline confidence, rurality, and volume were not associated with post-implementation confidence, nor was post-implementation confidence associated with measurements of site performance.

Conclusion: Higher ratings of site organizational culture was associated with higher postimplementation confidence in providing stroke care. This suggests that an individual site's context is a larger driver of the confidence in program implementation than volume or program performance. In addition, the finding that sites with longer time spent in the NTSP had lower post-implementation confidence suggests sites may benefit from periodic retraining to sustain confidence in their ability to provide stroke care. 